\title{
Ceruloplasmin and Iron Proteins in the Serum of Patients with Alzheimer's Disease
}

\author{
Gudlaug Torsdottir a, b Jakob Kristinsson ${ }^{a}$ Jón Snaedal ${ }^{b}$ \\ Torkell Jóhannesson ${ }^{\mathrm{a}}$ \\ ${ }^{a}$ Department of Pharmacology and Toxicology, University of Iceland, and ${ }^{b}$ Department of \\ Geriatrics, Landspítali-University Hospital, Reykjavík, Iceland
}

\section{Key Words}

Alzheimer's disease $\cdot$ Ceruloplasmin $\cdot$ Ferritin $\cdot$ Iron • Oxidative activity $\cdot$ Transferrin

\begin{abstract}
Backgrounds/Aims: The oxidative activity of ceruloplasmin (CP) in serum has been found to be lowered in patients with Alzheimer's disease (AD). We investigated whether changes in CP were reflected by altered iron parameters in AD patients. Methods: Iron parameters, and CP concentration, activity and specific activity were determined in the serum of 41 AD patients and controls. Results: $\mathrm{CP}$ activity and specific activity were significantly lower in the $A D$ patients. $C P$ concentration and activity were negatively correlated with the ferritin concentration in both groups. CP concentration was positively correlated with age in the control group but not in the patients group. Conclusion: The lowered CP activity in the serum of AD patients was not reflected by the iron parameters. As CP concentration only rises with age in the controls, this may indicate failing adaption to age-related alterations in iron metabolism in AD patients.
\end{abstract}

Copyright $\odot 2011$ S. Karger AG, Basel

\section{Introduction}

Iron accumulates in the brain with normal aging [1]. On the other hand, several authors have reported dysregulation of iron metabolism and oxidative stress due to redox-active iron in the brains of patients with Alzheimer's disease (AD) and other neurodegenerative diseas- 
Table 1. Characteristics of the study patients

\begin{tabular}{|c|c|}
\hline \multicolumn{2}{|c|}{ Dement Geriatr Cogn Disord Extra 2011;1:366-371 } \\
\hline $\begin{array}{l}\text { DOI: 10.1159/000330467 } \\
\text { Published online: November 5, } 2011\end{array}$ & $\begin{array}{l}\text { ( ) } 2011 \text { S. Karger AG, Basel } \\
\text { www.karger.com/dee }\end{array}$ \\
\hline
\end{tabular}

Patients (males/females), $\mathrm{n}$

Mean age (range), years

Mean MMSE score (range)
$41(18 / 23)$

$80(65-88)$

$18(6-27)$

MMSE scores <24 indicate dementia; 34 patients were examined.

es $[2,3]$. Disturbances in iron metabolism may occur at several stages. Ceruloplasmin (CP) is crucial in the oxidation of $\mathrm{Fe}^{2+}$ to $\mathrm{Fe}^{3+}$, which enables the binding of iron ions to transferrin and thereby the transport of iron throughout the body. Increased lipid peroxidation and iron deposition have been found in the brain of patients with aceruloplasminemia $[4,5]$. These patients had raised serum ferritin, indicating iron overload, but low transferrin saturation and mild anemia, which reflects the interaction between these three proteins. Furthermore, they suffered from progressive neurological disorders, including extrapyramidal disorders and dementia. Transferrin is to a large extent responsible for the transfer of iron in the body. The genetic variant of transferrin, subtype $\mathrm{C} 2$, has been investigated as potential risk factor for AD but the results were controversial [6, 7]. A recent study reported an altered ratio between $\mathrm{CP}$ and transferrin in the serum of $\mathrm{AD}$ patients compared with healthy individuals, and this ratio was inversely correlated with Mini Mental State Examination (MMSE) scores [8]. Ferritin is the main protein involved in iron storage. It accumulates in the brain as a result of normal aging, but differences in ferritin distribution in the brain between AD patients and controls have also been described [2].

In a case-control study by Snaedal et al. [9] in 1998, CP activity, but not CP concentration, was significantly lower in AD patients compared with controls. The aim of the present study was to verify the previously published results and investigate whether lowered CP activity in the serum of AD patients reflects to a significant degree changes in the serum levels of iron, transferrin and ferritin, and transferrin saturation in these patients.

\section{Patients and Methods}

\section{Patients}

Forty-one AD patients were included in the study. All patients had been examined at a psychogeriatric outpatient clinic and diagnosed with probable dementia of the Alzheimer's type according to the NINCS/ADRA criteria [10]. Their mean MMSE score was 18 (34 patients) and their mean age was 80 years (table 1). All patients were living in their homes but attending day-care centers specialized in caring for people with dementia for social and medical support. Patients with a serious medical condition or taking drugs known to affect either liver or kidney function were excluded. Each patient had an age- and gender-matched control randomly selected from the Icelandic National Registry. All persons in the control group were healthy and living independently in their homes. Exclusion criteria for the controls included a history of neurodegenerative disease, serious medical conditions or current drug treatment known to affect either liver or kidney function. $\gamma$ Glutamyl transpeptidase (GGT) was used as a marker for liver disease in the patients and the controls. Individuals exceeding twice the upper limits of GGT in healthy subjects were excluded from the study. 
Table 2. Mean CP, iron, ferritin and transferrin concentrations, CP oxidative activity, CP-specific oxidative activity and transferrin saturation in $\mathrm{AD}$ patients and their gender- and age-matched controls

\begin{tabular}{lcccc}
\hline Determinations in serum & Patients & Controls & Pairs, $\mathrm{n}$ & $\mathrm{p}$ value \\
\hline Serum CP oxidative activity, U/ml & 119 & 136 & 41 & 0.0165 \\
CP-specific oxidative activity, U/mg & 568 & 611 & 40 & 0.0040 \\
Concentrations & & & & \\
$\quad$ CP, $\mathrm{mg} / \mathrm{l}$ & 226 & 223 & 40 & 0.8501 \\
$\quad$ Iron, $\mu \mathrm{mol} / \mathrm{l}$ & 15 & 18 & 41 & 0.0828 \\
$\quad$ Ferritin, $\mu \mathrm{g} / \mathrm{l}$ & 114 & 2.4 & 40 & 0.2551 \\
$\quad$ Transferrin, g/l & 2.5 & 60 & 41 & 0.3011 \\
Transferrin saturation, $\mu \mathrm{mol} / \mathrm{l}$ & 61 & & 41 & 0.4808 \\
\hline
\end{tabular}

The number of pairs used for each analysis is indicated. The null hypothesis was rejected for $\mathrm{p} \leq 0.05$. The Mann-Whitney test was employed.

\section{Methods}

Fasting blood samples from patients and controls were obtained in the morning. The same blood sampling procedure was used for both groups, and the blood sampling units used were specially prepared for metal analysis (Sarstédt ${ }^{\circledR}$ ).

The study was approved by the Icelandic National Bioethics Committee (04-121) and registered by the Data Protection Authority.

GGT. Serum GGT activity (U/l) was determined using a Vitros 950 (Ortho-Clinical Diagnostics, Rochester, N.Y., USA) at the Icelandic University Hospital Laboratories.

$\mathrm{CP}$ Concentration. Serum CP levels $(\mathrm{mg} / \mathrm{l})$ were determined using a rate nephelometry immunoassay (ICS-2; Beckman Instruments Inc., Fullerton, Calif., USA) at the Icelandic University Hospital Laboratories. The results are presented as milligrams per liter in table 2.

Oxidative Activity of CP in the Serum. Oxidative activity of CP in the serum was determined at the Department of Pharmacology and Toxicology, University of Iceland, using the manual kinetic assay first described by Boyett et al. [11] in 1976 and later on by Transdottir et al. [12]. The results are presented as units per milliliter in table 2.

Serum Transferrin, Total Iron Binding Capacity and Transferrin Saturation. Serum transferrin $(\mathrm{mg} / \mathrm{l})$ was determined at the Icelandic University Hospital Laboratories. It was measured with an immunoturbidimetric assay on a Hitachi 911 (Roche, Basel, Switzerland). Total iron binding capacity was measured on a Vitros 5.1 from Ortho-Clinical Diagnostics (results not shown). Transferrin saturation $(\mu \mathrm{mol} / \mathrm{l})$ was calculated as follows: $(100 \times$ serum iron)/total iron binding capacity. The results are presented in table 2.

Serum Iron. Serum iron was determined at the Icelandic University Hospital Laboratories. It was also measured using a Vitros 5.1. The results are presented as micromoles per liter in table 2.

Serum Ferritin. Serum ferritin was determined at the Icelandic University Hospital Laboratories. It was assessed using an electrochemiluminescence immunoassay (E-Modular) from Roche. The results are presented as micrograms per liter in table 2.

\section{Statistical Analysis}

As the results for the specific activity of $\mathrm{CP}$ and ferritin concentration in serum did not pass a normality test, the results shown in table 2 were analyzed using the Mann-Whitney test. All the other determinations were also tested with Student's t test (results not shown), but this did not change the significance of the results presented in table 2. For these calcula- 
tions GraphPad Prism ${ }^{\circledR}$ was used. In this program, normality is calculated with both the Kolmogorov-Smirnov test and the newer D'Agostino-Pearson omnibus and Shapiro-Wilk tests. Linear regression was used to examine the correlation of age with ferritin, transferrin, $\mathrm{CP}$ concentration and $\mathrm{CP}$ activity. Furthermore, the correlation between $\mathrm{CP}$ concentration and $\mathrm{CP}$ activity was examined. Finally, the correlations between $\mathrm{CP}$ concentration and the concentration of ferritin and transferrin, respectively, were examined. Regression analyses were done with MedCalc ${ }^{\circledR}$.

\section{Results}

The results of $\mathrm{CP}$ oxidative activity, $\mathrm{CP}$-specific oxidative activity, concentrations of $\mathrm{CP}$, iron, transferrin and ferritin, and transferrin saturation in $\mathrm{AD}$ patients and their gender- and age-matched controls are shown in table 2 . CP concentration was positively correlated with age in the control group $(\mathrm{p}=0.007)$, but it was negatively correlated with ferritin concentration in patients and controls ( $\mathrm{p}=0.004$ and 0.006 , respectively). CP activity was negatively correlated with the ferritin concentration in patients and controls $(\mathrm{p}=0.042$ and 0.001 , respectively).

\section{Discussion}

This study examined the concentration, activity and specific activity of $\mathrm{CP}$ in $\mathrm{AD}$ patients and healthy controls. No difference in CP concentration was found between the AD patients and the controls, but CP activity and specific activity (activity divided by mass) was significantly lower in the AD patients. In the study by Snaedal et al. [9], CP concentration was normal but $\mathrm{CP}$ activity was decreased in $\mathrm{AD}$ patients compared with controls, which is in agreement with our results and further supported by the results of Brewer et al. [13] published in 2010, who found no difference in CP concentration between AD patients and controls, but circulating $\mathrm{CP}$ without oxidative activity was increased in AD patients. On the other hand, in a study by Squitti et al. [14], CP concentration was raised in the serum of AD patients. However, subsequently, the same authors found fragments of CP proteolysis in the serum of $\mathrm{AD}$ patients, possibly indicating impaired CP activity [15]. In the plasma of patients with aberrant levels of amyloid $\beta_{42}$, tau and phosphorylated tau in the CSF, CP levels were decreased compared with AD patients with normal levels of these biomarkers in the CSF [16]. Interestingly, $\mathrm{CP}$ has been found to be increased in the brain of $\mathrm{AD}$ patients, but only moderately changed in neurons, and it was not related to the redox-active iron found in neurofibrillary tangles, indicating a failure in the response of neural induction of $\mathrm{CP}$, resulting in the excessive accumulation of redox-active iron in the brain of AD patients [17]. It is still not known whether there is a connection between these changes in the brain and the changes in AD blood found in the above studies.

It seems that the patients in the present study (table 1), the study by Brewer et al. [13] and the patients with CSF-confirmed AD in the study by Kessler et al. [16] are older and/or with more progressive AD than the patients in the study by Squitti et al. [15] (2008). This may explain the discrepancy between the results of these studies and raises the question of whether early changes in $\mathrm{CP}$ are present in younger individuals who will later develop $\mathrm{AD}$. It is possible that $\mathrm{CP}$ is increased in younger $\mathrm{AD}$ patients to compensate for increased oxidative load, but with progressing disease, the patients lose this ability to compensate, and CP activity thus declines. The fact that CP concentration was positively correlated with age in the controls but not in the $\mathrm{AD}$ patients may furthermore reflect a loss of compensation for oxidative stress 
in progressing $\mathrm{AD}$. This is in line with the results of Arnal et al. [3], who found plasma $\mathrm{CP}$ concentration to be lower in the latest stage of $\mathrm{AD}$ compared to earlier stages and that in healthy elderly controls [18]. Another possibility is that a genetic defect in CP is a risk factor for $\mathrm{AD}$, as has been suggested for patients with Parkinson's disease. We calculated the specific activity of CP (activity/mass) and found it to be significantly lower in the blood of the $\mathrm{AD}$ patients than in the control group, most likely indicating a poorer quality of the $\mathrm{CP}$ protein in the AD patients. This is in line with results of studies on patients with Parkinson's disease, in whom iron accumulation has been established in the substantia nigra along with genetic defects in CP $[3,12,19]$. Taken together, these results may indicate that AD is a multifactorial disease with several risk factors, and that $\mathrm{CP}$ dysfunction resulting in oxidative stress may be one of the contributing factors, although it remains to be established whether it is caused by a genetic defect or acquired later in life.

There was no significant difference in the iron and iron protein results between the AD patients and the controls (table 2). This is in agreement with earlier work by Fischer et al. [20], who did not find a difference in the ferritin or transferrin concentration between AD patients and controls. However, our study showed a positive correlation between age and CP concentration in the controls, which was absent in the patients. Furthermore, CP concentration and activity were negatively correlated with ferritin concentration in both the patients and the controls, reflecting an inverse relationship between $\mathrm{CP}$ and ferritin. This may indicate an increased iron load in the tissue of $\mathrm{AD}$ patients compared with the controls. Ferritin is an intracellular iron storage protein, consisting of two subunits (a large and a small one) in different ratios in different tissues. The large subunit has ferroxidase activity, while the small subunit is associated with the mineralization of iron at the ferritin core [21]. The ratio of heavy to light chains and the distribution of ferritin as well as the distribution of $\mathrm{CP}$ differ between $\mathrm{AD}$ patients and controls $[17,22]$. This raises the question of whether or not the ferroxidase activity of the larger subunits of ferritin and CP in the brain are related, and how disrupted interaction between the proteins may reflect the pathogenesis of AD.

In the present study, we found no relationship between $\mathrm{CP}$ and transferrin, and no difference in transferrin concentration or saturation between the AD patients and the controls. Transferrin has not been studied in detail in the blood of AD patients. However, Squitti et al. [8] found an altered $\mathrm{CP} /$ transferrin ratio between $\mathrm{AD}$ patients and controls, which was inversely correlated with the MMSE scores in AD patients. This is not in line with our results, and further studies are needed on the subject.

In conclusion, there is evidence of disrupted regulation of iron and oxidative stress in the brain of $\mathrm{AD}$ patients. CP plays a central role in iron handling and is altered in both the brain and the blood of $\mathrm{AD}$ patients. In this study, $\mathrm{CP}$ activity and specific activity were significantly lower in the $\mathrm{AD}$ patients compared with the controls. We found a negative correlation between CP concentration/activity and ferritin concentration in the blood of both groups, indicating interplay between these proteins. However, as $\mathrm{CP}$ concentration fails to increase in $\mathrm{AD}$ patients with age, this may aggravate an age-related iron dysregulation in $\mathrm{AD}$ patients.

\section{References}

-1 Zecca L, Youdim MB, Riederer P, Connor JR, Crichton RR: Iron, brain ageing and neurodegenerative disorders. Nat Rev Neurosci 2004;5:863-873.

2 Quintana C, Bellefqih S, Laval JY, Guerquin-Kern JL, Wu TD, Avila J, et al: Study of the localization of iron, ferritin, and hemosiderin in Alzheimer's disease hippocampus by analytical microscopy at the subcellular level. J Struct Biol 2006;153:42-54. 
-3 Hochstrasser H, Bauer P, Walter U, Behnke S, Spiegel J, Csoti I, et al: Ceruloplasmin gene variations and substantia nigra hyperechogenicity in Parkinson disease. Neurology 2004;63:1912-1917.

-4 Yoshida K, Kaneko K, Miyajima H, Tokuda T, Nakamura A, Kato M, et al: Increased lipid peroxidation in the brains of aceruloplasminemia patients. J Neurol Sci 2000;175:91-95.

-5 Xu X, Pin S, Gathinji M, Fuchs R, Harris ZL: Aceruloplasminemia: an inherited neurodegenerative disease with impairment of iron homeostasis. Ann NY Acad Sci 2004;1012:299-305.

-6 Robson KJ: Synergy between the C2 allele of transferrin and the C282Y allele of the haemochromatosis gene (HFE) as risk factors for developing Alzheimer's disease. J Med Genet 2004;41:261-265.

-7 van Rensburg SJ, Potocnik FC, De Villiers JN, Kotze MJ, Taljaard JJ: Earlier age of onset of Alzheimer's disease in patients with both the transferrin C2 and apolipoprotein E-epsilon 4 alleles. Ann NY Acad Sci 2000;903:200-203.

8 Squitti R, Salustri C, Siotto M, Ventriglia M, Vernieri F, Lupoi D, et al: Ceruloplasmin/transferrin ratio changes in Alzheimer's disease. Int J Alzheimers Dis 2010;2011:231595.

-9 Snaedal J, Kristinsson J, Gunnarsdottir S, Olafsdottir A, Baldvinsson M, Johannesson T: Copper, ceruloplasmin and superoxide dismutase in patients with Alzheimer's disease. A case-control study. Dement Geriatr Cogn Disord 1998;9:239-242.

-10 McKhann G, Drachman D, Folstein M, Katzman R, Price D, Stadlan EM: Clinical diagnosis of Alzheimer's disease: report of the NINCDS-ADRDA Work Group under the auspices of Department of Health and Human Services Task Force on Alzheimer's Disease. Neurology 1984;34:939-944.

-11 Boyett JD, Lehmann HP, Beeler MF: Automated assay of ceruloplasmin by kinetic analysis of o-dianisidine oxidation. Clin Chim Acta 1976;69:233-241.

-12 Torsdottir G, Kristinsson J, Sveinbjornsdottir S, Snaedal J, Johannesson T: Copper, ceruloplasmin, superoxide dismutase and iron parameters in Parkinson's disease. Pharmacol Toxicol 1999;85:239243.

-13 Brewer GJ, Kanzer SH, Zimmerman EA, Celmins DF, Heckman SM, Dick R: Copper and ceruloplasmin abnormalities in Alzheimer's disease. Am J Alzheimers Dis Other Demen 2010;25:490-497.

-14 Squitti R, Pasqualetti P, Dal Forno G, Moffa F, Cassetta E, Lupoi D, et al: Excess of serum copper not related to ceruloplasmin in Alzheimer disease. Neurology 2005;64:1040-1046.

-15 Squitti R, Quattrocchi CC, Salustri C, Rossini PM: Ceruloplasmin fragmentation is implicated in 'free' copper deregulation of Alzheimer's disease. Prion 2008;2:23-27.

-16 Kessler H, Pajonk FG, Meisser P, Schneider-Axmann T, Hoffmann KH, Supprian T, et al: Cerebrospinal fluid diagnostic markers correlate with lower plasma copper and ceruloplasmin in patients with Alzheimer's disease. J Neural Trasm 2006;113:1763-1769.

- 17 Castellani RJ, Smith MA, Nunomura A, Harris PL, Perry G: Is increased redox-active iron in Alzheimer disease a failure of the copper-binding protein ceruloplasmin? Free Radic Biol Med 1999;26: $1508-1512$.

-18 Arnal N, Cristalli DO, de Alaniz MJ, Marra CA: Clinical utility of copper, ceruloplasmin, and metallothionein plasma determinations in human neurodegenerative patients and their first-degree relatives. Brain Res 2010;1319:118-130.

-19 Jin L, Wang J, Zhao L, Jin H, Fei G, Zhang Y, et al: Decreased serum ceruloplasmin levels characteristically aggravate nigral iron deposition in Parkinson's disease. Brain 2011;134(Pt 1):50-58.

-20 Fischer P, Gotz ME, Danielczyk W, Gsell W, Riederer P: Blood transferrin and ferritin in Alzheimer's disease. Life Sci 1997;60:2273-2278.

-21 Arosio P, Levi S, Santambrogio P, Cozzi A, Luzzago A, Cesareni G, et al: Structural and functional studies of human ferritin H and L chains. Curr Stud Hematol Blood Transfus 1991;58:127-131.

-22 Connor JR, Menzies SL: Cellular management of iron in the brain. J Neurol Sci 1995;134(suppl): $33-44$. 